\title{
RAD tag sequencing as a source of SNP markers in Cynara cardunculus L
}

Davide Scaglione ${ }^{1}$, Alberto Acquadro ${ }^{1}$, Ezio Portis ${ }^{1}$, Matteo Tirone ${ }^{1}$, Steven J Knapp ${ }^{2}$ and Sergio Lanteri ${ }^{{ }^{*}}$

\begin{abstract}
Background: The globe artichoke (Cynara cardunculus L. var. scolymus) genome is relatively poorly explored, especially compared to those of the other major Asteraceae crops sunflower and lettuce. No SNP markers are in the public domain. We have combined the recently developed restriction-site associated DNA (RAD) approach with the Illumina DNA sequencing platform to effect the rapid and mass discovery of SNP markers for C. cardunculus.

Results: RAD tags were sequenced from the genomic DNA of three C. cardunculus mapping population parents, generating 9.7 million reads, corresponding to $\sim 1 \mathrm{Gbp}$ of sequence. An assembly based on paired ends produced $\sim 6.0 \mathrm{Mbp}$ of genomic sequence, separated into 19,000 contigs (mean length $312 \mathrm{bp}$ ), of which $21 \%$ were fragments of putative coding sequence. The shared sequences allowed for the discovery of $\sim 34,000$ SNPs and nearly 800 indels, equivalent to a SNP frequency of 5.6 per 1,000 nt, and an indel frequency of 0.2 per 1,000 nt. A sample of heterozygous SNP loci was mapped by CAPS assays and this exercise provided validation of our mining criteria. The repetitive fraction of the genome had a high representation of retrotransposon sequence, followed by simple repeats, AT-low complexity regions and mobile DNA elements. The genomic k-mers distribution and CpG rate of $C$. cardunculus, compared with data derived from three whole genome-sequenced dicots species, provided a further evidence of the random representation of the $C$. cardunculus genome generated by RAD sampling.

Conclusion: The RAD tag sequencing approach is a cost-effective and rapid method to develop SNP markers in a highly heterozygous species. Our approach permitted to generate a large and robust SNP datasets by the adoption of optimized filtering criteria.
\end{abstract}

\section{Background}

Cynara cardunculus $(2 n=2 x=34$, haploid genome size $\sim 1.08 \mathrm{Gbp}[1])$ an allogamous, highly heterozygous Asteraceae species, includes three taxa: the globe artichoke (var. scolymus), the cultivated cardoon (var. altilis) and their common progenitor the wild cardoon (var. sylvestris) [2]. Globe artichoke contributes significantly to the Mediterranean agricultural economy, and is also cultivated in South America, North Africa, China and USA. Over the past 30 years, a body of evidence has grown that plantbased foods can be effective for the alleviation of several chronic diseases, and globe artichoke in particular has been shown to produce a number of nutraceutically and pharmaceutically active compounds. Extracts from both globe artichoke and cultivated cardoon have exhibited

\footnotetext{
* Correspondence: sergio.lanteri@unito.it

'Di.Va.P.R.A. Plant Genetics and Breeding, University of Torino, via L. da Vinci 44, 10095 Grugliasco (Torino), Italy

Full list of author information is available at the end of the article
}

hepatoprotective, anticarcinogenic, antioxidative and antibacterial qualities, and even an inhibition of cholesterol biosynthesis and LDL oxidation [3-6]. Finally, there is increasing interest in developing the species as an energy and oilseed crop [7-10].

Since the first linkage map produced for globe artichoke [11], a number of other segregating populations have been exploited for genetic mapping, including one generated from a hybrid between a globe artichoke and a cultivated cardoon genotype [12] and, more recently, one obtained by crossing globe artichoke with wild cardoon [13]. The recent development of a set of gene-based microsatellites [14] has aided the construction of consensus genetic maps $[13,15,16]$. However, these maps remains insufficiently densely populated for trait mapping and marker assisted selection. Current high throughput sequencing technology, which produces DNA sequence at a rate several orders of magnitude faster than conventional methods, is effective as a platform for SNP (single nucleotide polymorphism)
C Biomed Central

() 2012 Scaglione et al; licensee BioMed Central Ltd. This is an Open Access article distributed under the terms of the Creative Commons Attribution License (http://creativecommons.org/licenses/by/2.0), which permits unrestricted use, distribution, and reproduction in any medium, provided the original work is properly cited. 
discovery. A particularly efficient protocol, termed "restriction-site associated DNA" (RAD) [17], in combination with the Illumina Genome Analyzer sequencing device [18], discovers SNPs by sequencing a large set of restriction fragments [19-21]. Here we report the generation of genomic RAD tags from the three $C$. cardunculus accessions used as the parents for two of our mapping populations. The RAD tags were used to derive SNP markers some of which were then validated by a Cleaved Amplified Polymorphic Sequence (CAPS) assay. The identified SNPs could be useful to produce denser $C$. cardunculus genetic maps via high-throughput genotyping technologies. The RAD sequence has also been informative for characterizing the repetitive DNA component of the C. cardunculus genome, in particular allowing some inferences to be made regarding the contribution of DNA methylation in inhibiting its expansion.

\section{Results and Discussion}

\section{RAD tag sequencing and de novo contig assembly}

The sequencing of the RAD libraries obtained from the three $C$. cardunculus accessions generated some 9.7 million reads (19.4 million paired ends), corresponding to $\sim 1$ Gbp of sequence. As reported previously [22], the distribution of reads was non-uniform across the three DNA samples, with 1.2 million reads achieved for globe artichoke, 2.6 million for cultivated cardoon and 5.9 million for wild cardoon. As a result, the wild cardoon variety was chosen as the basis for de novo contigs assembly. The sequence assembly pipeline (Figure 1 ) generated 19,061 reference contigs (Additional file 1), spanning 6.11 Mbp. The GC content of the sequence was about $37.4 \%$, close to that prevailing in both Arabidopsis thaliana [23] and Vitis vinifera [24].

As expected from the size-selection procedure used in the construction of the libraries, N50 was 321 bp and the mean contigs length was 312 bp (Figure 2). The reported contig length distribution is similar to the one described by Etter et al. [25], while other research (Baxter et al. [26], Willing et al. [27]) reported RAD contig lengths skewed towards the longer fragments. We hypothesize these differences to be related to coverage depth obtained during sequencing, as we used for our assembly $\sim 6 \mathrm{M}$ total reads, while Etter et al. used $\sim 8 \mathrm{M}$ reads, Baxter et al. $\sim 13 \mathrm{M}$ reads, and Willing et al. $\sim 23 \mathrm{M}$ reads. Furthermore, for the generation of RAD sites, we used a 6-cutter (PstI) enzyme while Etter et al. [25] and Baxter et al. [26] used SbfI, which is an 8-cutter. By targeting a reduced amount of genomic loci it's likely to gain a relative higher coverage which can promote the assembly of longer contigs. Alternative assemblies (i.e. more than one contig generated per RAD site, see "Materials and methods") accounted for less than the $7 \%$ of the RAD contig set, similarly to what reported by Willing et al. [27].

\section{Annotation and GO categorization of contigs}

The BLASTX search resulted in a top-hit list (composed by the first result of each BLAST output report) of protein sequences from $V$. vinifera ( $41 \%$ of the total hits), Ricinus communis (16\%), Populus trichocarpa (15\%) and A. thaliana (6\%). Gene Ontology (GO) terms were assigned to 3,791 contigs (19.8\%; Figure 3, Additional file 2). Most of the failed annotations (72.0\%) applied to contigs lacking any BLASTX hit; of the remainder, $5.8 \%$ did not pass the annotation threshold and $2.3 \%$ resulted in no GO mapping. Overall, 5,335 contigs (28.0\%) included at least one BLASTX hit with an E-value $<10 \mathrm{e}^{-3}$, with 3,554 of these (18.6\%) recording an E-value $<10 \mathrm{e}^{-15}$. Despite the genome-wide RAD sampling, a noteworthy part of it may be likely represented by coding regions, since a methylationsensitive enzyme (PstI) was used to produce the RAD-tag libraries [28]; notwithstanding the rather short length of the RAD contigs made it difficult to distinguish between sequences representing complete genes and pseudogenes. Enzyme codes were retrieved for 1,327 contigs, defining a unique set of 313 putative enzymatic activities, which were mapped onto KEGG reference pathways (Additional file $3)$. Within the repetitive DNA fraction (Figure 4 ), 1.2\% of the sequences were derived from LTR retroelements, including Ty/Copia-like (0.8\%) and Gypsy-like (0.2\%). Transposable DNA element footprints accounted for a further $0.2 \%$ of the sequence. Note that this quantification of transposable element abundance could have been underestimated by the shortness of the RAD tag sequences which could affect search sensitivity.

\section{K-mer distribution analysis}

With the aim to investigate whether the RAD sequencing was able to provide a representative and unbiased sample of the C. cardunculus genome, we compared the k-mers spectrum with other fully sequenced genomes. Moreover, we further investigated how CpG content correlate with the repetitive contents of the genome, as suggested by Chor et al. [29]. The frequency and distribution of 10-mers among the raw sequence and the assembled wild cardoon contigs were comparable to one another (Figure 5A). $\mathrm{K}$-mers lacking $\mathrm{CpG}$ dinucleotides were over-represented in the more repetitive portion of the spectra (i.e. their distribution was right-skewed), while those bearing at least one CpG produced a more left-shifted distribution (Figure 5A). Results were confirmed by negative controls through the adoption of random dinucleotides, which did not show any preferential distributions of K-mers (Additional file 4). This outcome is consistent with the known correlation of $\mathrm{CpG}$ methylation with the repression of transposable elements [30,31]. A comparative study of other plant genomes showed that the $V$. vinifera genome has a higher frequency of zero-CpG K-mers (Figure $5 \mathrm{C}$ ) than that of $A$. thaliana (Figure $5 \mathrm{~B}$ ), but that the Fragaria 


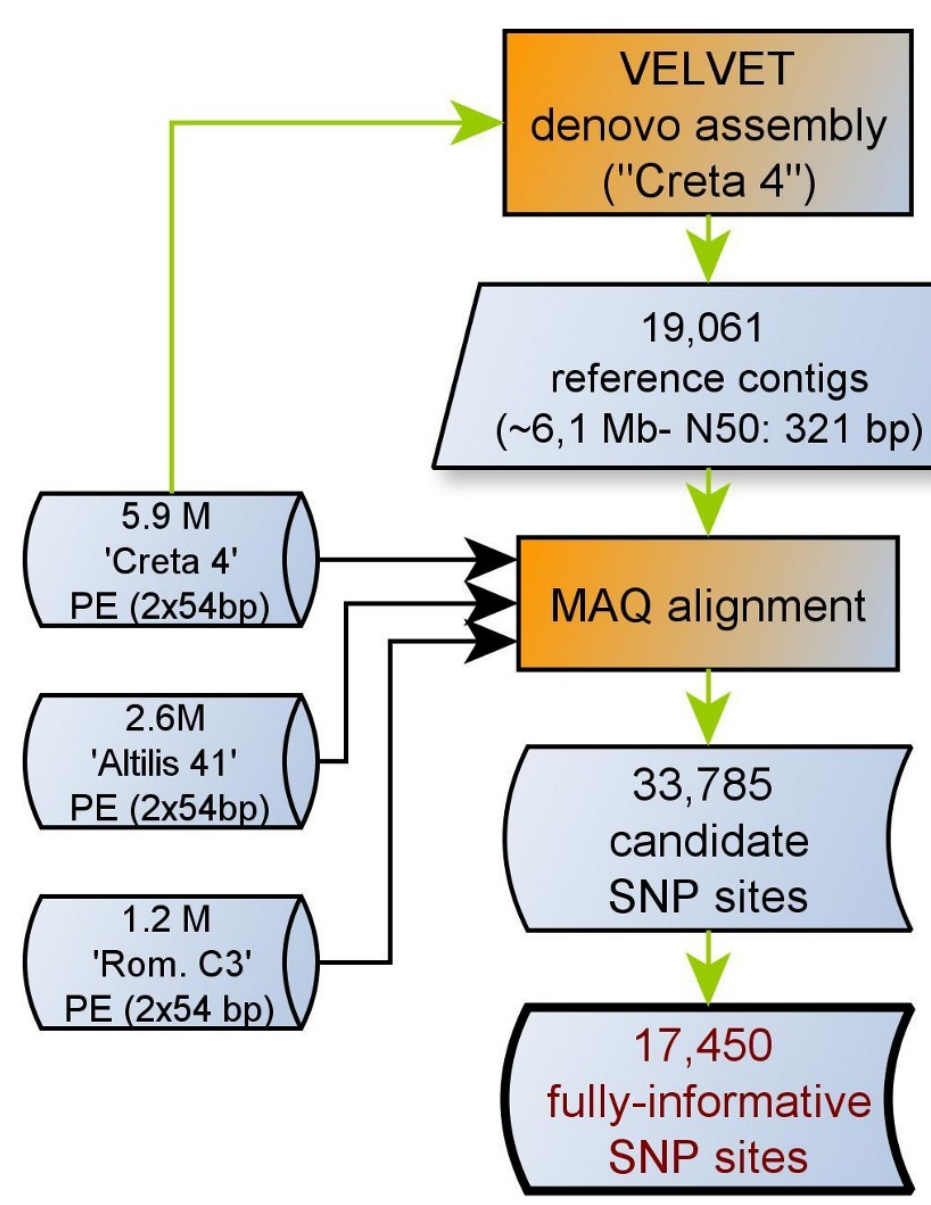

Figure 1 Contig assembly and SNP discovery. The de novo assembly was based on paired ends from "Creta 4". The alignment of paired ends was used to discover SNPs, using MAQ software. "Fully informative" SNP sites were those where sequence information was available for all three parental accessions.

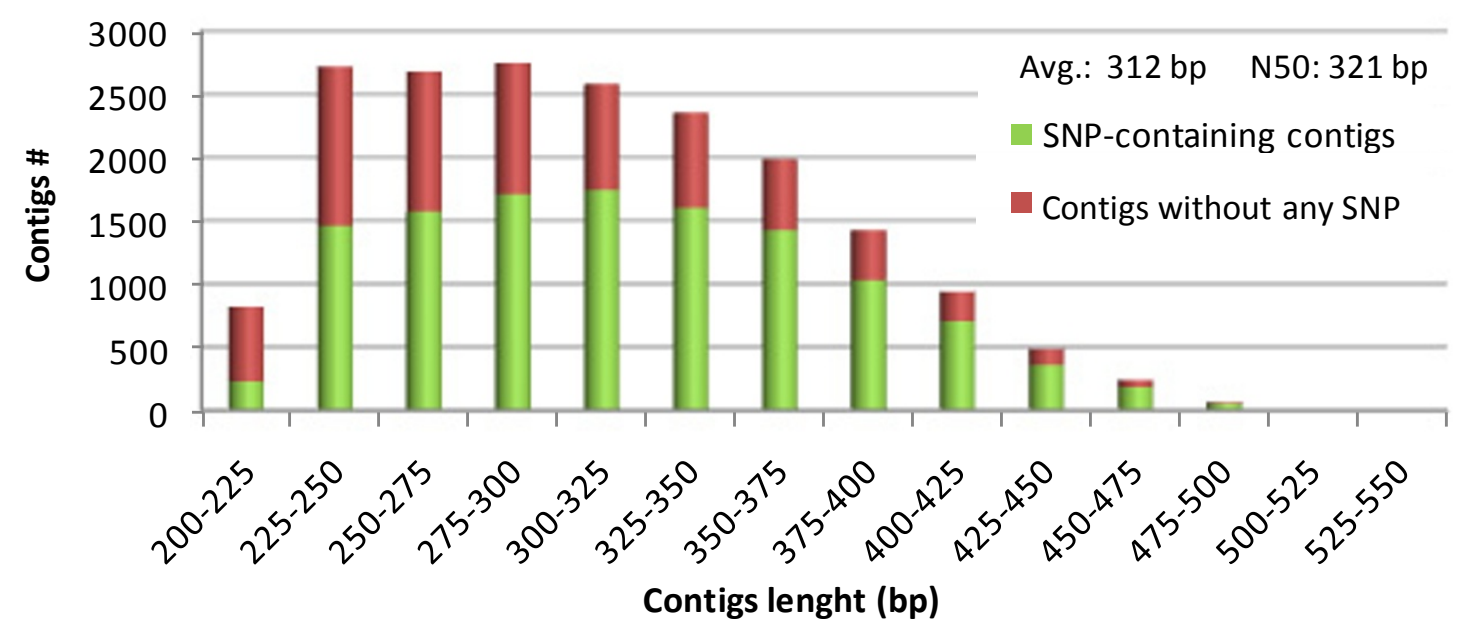

Figure 2 Contig length distribution and the efficiency of SNP discovery. Red bars represent the portion of contigs having no SNP identified, while green bars represent contigs harbouring at least one SNP. 


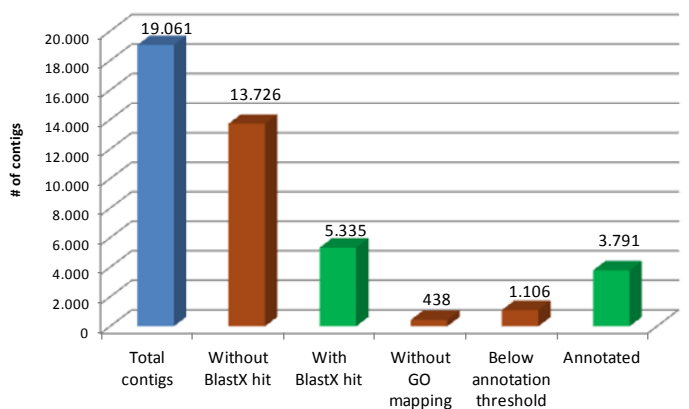

Figure 3 Contig annotation. Green bars represent sequences which either produced a BLASTX hit or passed the final annotation criteria. Brown bars represent contigs filtered out because of an absence of a BLASTX hit, no GO mapping or an annotation score below the threshold.

vesca $\mathrm{K}$-mer distribution (Figure 5D) was rather similar to that obtained in C. cardunculus (Figure 5A). To futher investigate these trends, CpG rates [32] across the four dicot species were compared. While the CpG rate in the $C$. cardunculus RAD dataset was 0.53 , 0.72 was calculated for $A$. thaliana, 0.43 for $V$. vinifera and 0.61 for $F$. vesca genomes [23,24,33]. Furthermore, the A. thaliana genome includes a $14 \%$ presence of repetitive elements [23], that in $V$. vinifera is $41 \%$ [24], and that in F. vesca $22 \%$ [33]. Variations in CpG rates showed to be congruent with data derived from $\mathrm{K}$-mer spectra analysis, since genomes harbouring higher rates of $\mathrm{CpG}$ reported less repetitive K-mer populations. This suggests a key contribution of DNA methylation in the inhibition of genome expansion due to repetitive element proliferation.

Altogether, our data suggest that the RAD procedure, despite its use of GC-rich recognition sites, has produced a random representation of the $C$. cardunculus genome, and shows that it represents a reliable means of assessing genome complexity.

\section{SNP calling and classification}

The paired ends generated for each mapping parent were aligned based on the reference contig set. This alignment detected 33,784 sequence variants, including 1,520 short indels, scattered over 12,068 contigs ('CcRAD1' dataset, Additional file 5). The overall SNP frequency was estimated to be 5.6 per 1,000 nucleotides, a level which is almost identical to that found in the non-coding regions of the $V$. vinifera genome (5.5 per 1,000 nucleotides) [34] and very similar to that uncovered among Citrus spp. ESTs (6.1 per 1,000 nucleotides) [35]. The estimation of SNP frequency using such high throughput sequencing data is, however, heavily dependent both on the number of genomes sampled, and on the extent (if any) of targeting and of genome coverage. The efficiency of SNP discovery was correlated with the length of the RAD tags (Figure 2). Contigs longer than 400 bp were associated with a $74 \%$ probability of finding at least one SNP, while this probability fell to $62 \%$ for contigs shorter than 400 bp. Setting as a criterion the need to identify SNPs informative for both mapping populations reduced the dataset

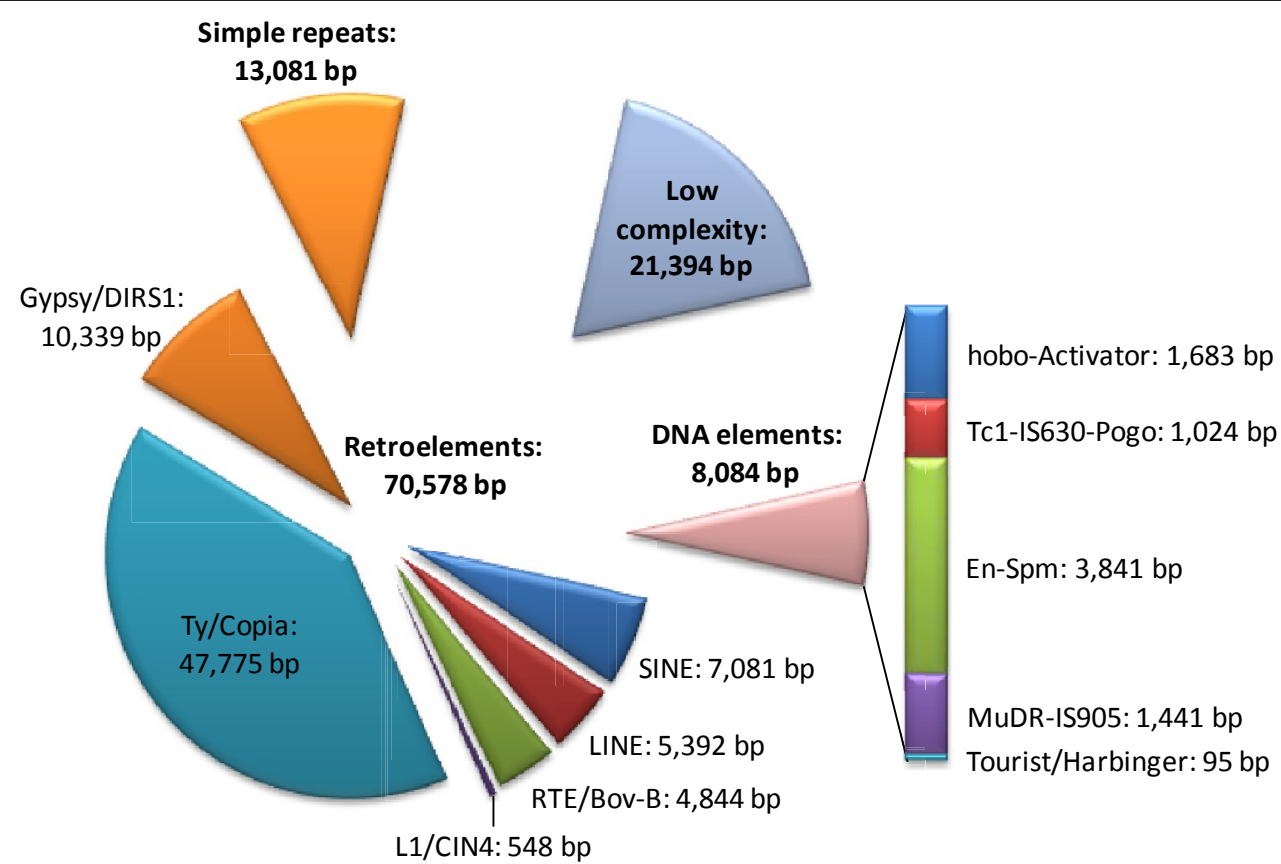

Figure 4 Repetitive DNA in the RAD contigs. The representation of known repetitive elements in C. cardunculus RAD sequence. Results generated by RepeatMasker analysis against the Repbase database. 


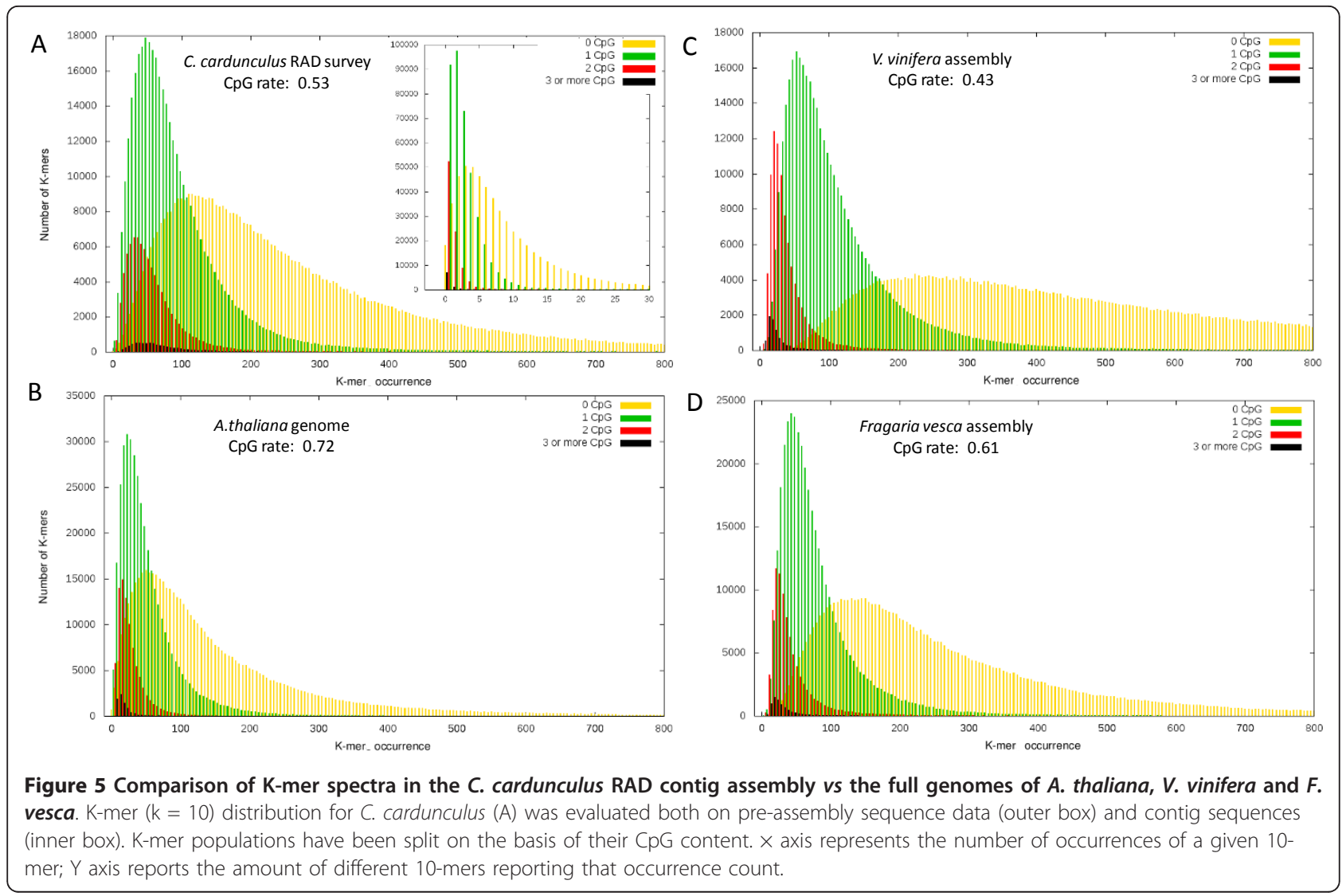

size to 17,450 sequence polymorphisms distributed over 7,478 contigs ('CcRAD2' dataset, Additional file 6); of these, 16,727 were SNPs, and 723 were 1 or 2 nt indels. Some $57 \%$ of the contigs contained more than one polymorphic site, and non bi-allelic variants occurred at 959 sites. The number of heterozygous SNP loci was 1,235 in the globe artichoke parent, 2,868 in the cultivated cardoon and 5,069 in the wild cardoon. The loci were classified into those expected to segregate in a 1:1 ratio ("testcross markers"), and those in a 1:2:1 ratio ("intercross markers") (Table 1, Additional file 6). The lower number of reads generated from the globe artichoke template produced an under-representation of testcross markers, compared to the levels of informativeness observed previously for other marker types [36]. Moreover, genetic diversity across the three taxa might be responsible for taxon-specific RAD tags due to the absence of PstI restriction sites. In the final dataset ("fully informative" SNP sites, Additional file 6), the proportion of contigs including more than one informative marker was $26 \%$.

\section{CAPS markers conversion and linkage analysis}

A random selection of 24 SNPs was made from the CcRAD2 dataset in order to validate the SNP calls by conversion to a CAPS format. These assays were then used to genotype the globe artichoke $\times$ cultivated cardoon mapping population members [12]. Primer pairs were designed for testcross SNP loci expected to segregate only within cultivated cardoon (Table 2). Successful amplification was obtained for all the assays, and 19 out of the 24 segregated consistently with the predicted 1:1

\section{Table 1 SNP mining results}

\begin{tabular}{llc}
\hline Filtering criteria & $\begin{array}{c}\text { RAD-contigs } \\
\text { count }\end{array}$ & $\begin{array}{c}\text { SNPs } \\
\text { count }\end{array}$ \\
\hline Total SNPs mining (CCRAD1) & 12,068 & 33,784 \\
"Fully informative" RAD loci (CcRAD2) & 7,478 & 17,450 \\
Putative testcross markers (CcRAD2) & 6,289 & 8,530 \\
$\quad$ "Romanesco C3" testcross over & 724 & 883 \\
$\quad$ "Altilis 41" & & \\
$\quad$ "Altilis 41" testcross over & 1,541 & 2,210 \\
$\quad$ "Romanesco C3" & & \\
$\quad$ "Romanesco C3" testcross over & 778 & 937 \\
$\quad$ "Creta 4" \\
$\quad$ C3" \\
Common intercross over "Romanesco & 3,246 & 4,500 \\
\hline
\end{tabular}

Two separated filtering criteria outcomes are reported (CCRAD1 and CCRAD2). Testcross and intercross markers evaluation was carried out exclusively on CCRAD2, representing SNP sites having sequence information for each of the three samples analyzed. 


\begin{tabular}{|c|c|c|c|c|c|c|c|c|c|c|}
\hline SNP-ID & Primer forward & Primer reverse & $\begin{array}{l}\text { Reads } \\
\text { ratio }\end{array}$ & Enzyme & $\begin{array}{l}\text { Product } \\
\text { size (bp) }\end{array}$ & $\begin{array}{l}\text { Restriction } \\
\text { site (bp) }\end{array}$ & $\begin{array}{l}\text { "RomanescoC3" } \\
\text { restriction produts }\end{array}$ & $\begin{array}{l}\text { "Altilis 41" } \\
\text { restriction } \\
\text { products }\end{array}$ & $\begin{array}{l}\text { Segregation } \\
\text { pattern }\end{array}$ & $\begin{array}{l}\text { Linkage } \\
\text { groups }\end{array}$ \\
\hline $211-167$ & TCAACCCAATCTCGTCAGTG & CTTCATAGTGGCAGCCTGGT & $10 / 30$ & ECORV & 372 & 162 & 372 & $372,210,162$ & Test cross & LG Alt_1a \\
\hline 4977-209 & AAATCCCACATATGGAAATAGC & TCATGACACAAGGTGGAGACA & $28 / 45$ & $X m n l$ & 360 & 176 & 360 & $360, \overline{176,184}$ & Test cross & LG Alt_2 \\
\hline $5548-175$ & AATGCACAAACCAAGTGCAA & TGAGCTCATTCGGAGGAAAT & $5 / 17$ & $X m n l$ & 248 & 110 & 138,110 & $\underline{248,138,110}$ & Test cross & $\begin{array}{l}\text { New LG } \\
\text { Alt_22 }\end{array}$ \\
\hline 5983-127 & TTGGTGGGTTTAAGACACCTIT & GTTAAACCCCCTGGATTGCT & $3 / 5$ & Taql & 179 & 118 & 179 & $179,61,118$ & Test cross & LG Alt_1b \\
\hline $\begin{array}{l}13671- \\
168\end{array}$ & TCTGGAGCATAAGAGGTAGGG & TTCAGTCGACTTCAAGGGAAC & $13 / 20$ & Fokl & 243 & 88 & 243 & $243,155,88$ & Test cross & LG Alt_1a \\
\hline $\begin{array}{l}14488- \\
152\end{array}$ & AAAGCTITITCCCCTITCC & AAGTGCGTATTTGATTGATTGA & $22 / 51$ & Msel & 388 & 150 & 388 & $388,238,150$ & Test cross & LG Alt_6 \\
\hline $\begin{array}{l}14600- \\
111\end{array}$ & AAAAACACGCTCCTTCCATA & TGTCATCCCCATGAAAAAGC & $7 / 12$ & $B c c l$ & 290 & 97 & 290 & $290,193,97$ & Test cross & $\begin{array}{l}\text { New } \\
\text { doublet }\end{array}$ \\
\hline $\begin{array}{l}20149- \\
154\end{array}$ & CCAGATGCAAATTGATACGTTG & GGATCTGCATTGAAACCTTGA & $10 / 21$ & ECORV & 262 & 153 & 153,109 & $\underline{264,153,109}$ & Test cross & LG Alt_1b \\
\hline $22767-99$ & CGGCACAACTAAGAGACAATCT & TTGGAGTATGTCTCGGGCTA & $8 / 15$ & BcCl & 315 & 88 & 315 & $315, \underline{227,88}$ & Test cross & LG Alt_18 \\
\hline $25124-86$ & ACAAGGCCGGACCCTAAAC & TGGAACAGGAAGGACAGGTT & $7 / 15$ & Dral & 288 & 71 & 288 & $288,217,71$ & Test cross & LG Alt_9 \\
\hline $\begin{array}{l}25294- \\
169\end{array}$ & GAGGAAACTTITCCCCATCG & CCGTTGTTGTATGCCTCAAA & $4 / 11$ & Xbal & 327 & 159 & 212,159 & $\underline{327,212,159}$ & Test cross & LG Alt_4 \\
\hline $\begin{array}{l}25584- \\
143\end{array}$ & ATTCGCCATGGAACAAGG & GCAGTCTAATGCTTCAACTGGT & $12 / 29$ & Taql & 272 & 89 & 183,89 & $\underline{272,183,79}$ & Unclear & - \\
\hline $\begin{array}{l}26480- \\
171\end{array}$ & CGACAAACTCCCTCCATGTT & TGTGGTATTGATGGGGAACC & $3 / 6$ & ECORV & 320 & 153 & 320 & $320,172,153$ & Test cross & LG Alt_2 \\
\hline $26420-81$ & ACATCAACGCCAGCAAAGAT & TTCTTGTTTGAATCTCAAGTGC & $5 / 18$ & $x m n l$ & 281 & 76 & 205,76 & $\underline{281,205,76}$ & Missing cut & - \\
\hline $\begin{array}{l}36002- \\
194\end{array}$ & GCACAGGAAAATGTTGGTGTTA & GTCTTTGCAATTCCAATCAGA & $5 / 16$ & Dral & 369 & 152 & 217,152 & $\underline{369,217,152}$ & Test cross & LG Alt_14 \\
\hline $\begin{array}{l}36199- \\
225\end{array}$ & TGACCAGGTTTCAGGTATGTG & AACGTACAAATTCAAAGCACGA & $7 / 11$ & $\mathrm{BamHI}$ & 398 & 221 & 221,177 & $\underline{398,221,177}$ & Test cross & LG Alt_8 \\
\hline $\begin{array}{l}38377- \\
214\end{array}$ & AGAACCCGAAAACGTCTCCA & AGGACCTAATGCAGGTTCTGA & $16 / 22$ & Ndel & 451 & 203 & 451 & $451,248,203$ & Test cross & LG Alt_4 \\
\hline $\begin{array}{l}38382- \\
111\end{array}$ & CAGGGAGAATCCCTCTCTCA & CATATATTGGATGATCCCTTGG & $4 / 9$ & Dral & 305 & 99 & 206,99 & $\underline{305}, 206,99$ & Unclear & - \\
\hline $40917-80$ & TGCTTCCCAATAGCCTCTAA & TGTGGTGATTITGGACGTGT & $7 / 13$ & Fokl & 306 & 70 & 306 & $306,236,7$ & Test cross & LG Alt_1a \\
\hline $43124-62$ & TGATTATGCATCACCCCAAA & CACTITAAATCCCAAAACAACC & $9 / 19$ & Taql & 309 & 52 & 257,52 & $\underline{309,257,52}$ & Test cross & LG Alt_4 \\
\hline $\begin{array}{l}43867- \\
147\end{array}$ & TGCATTTCTTCCTTGTGGTTC & ATGCTCCGTGAGGTTCGTAG & $10 / 19$ & ECORV & 314 & 138 & 176,138 & $\underline{316}, 176,138$ & Unclear & - \\
\hline $\begin{array}{l}45558- \\
111\end{array}$ & GGGAGAAGACCACGTAATTTGA & GTTTATTTCCGTCCCCAGGT & $10 / 19$ & Fokl & 294 & 122 & 172,122 & $\underline{294,172,122}$ & Test cross & LG Alt_5 \\
\hline $\begin{array}{l}45893- \\
190\end{array}$ & TCATTGGTCTTGCAGTTGGA & ACTTGGGCTGTAGCTTGACG & $8 / 13$ & Taql & 344 & 176 & 176,168 & $\underline{344}, 176,168$ & Test cross & LG Alt_18 \\
\hline $\begin{array}{l}45900- \\
239\end{array}$ & GGACAGTITTGAGAAATGGTCT & TCACACGGTITTGCAATCTC & $2 / 6$ & ECORV & 306 & 203 & 306 & $306,203,103$ & Missing cut & - \\
\hline
\end{tabular}


ratio (Table 2). Three of the assays produced not readable patterns of segregation and were discarded, while other two showed no evidence of any restriction cleavage, suggesting either a false SNP call (e.g. assembly of paralogs, sequencing error) or failure in the assay (e.g. selective amplification of one allele). Among the 19 CAPS loci retained, none showed a significant level of segregation distortion $\left(\chi^{2} \leq \chi_{\alpha=0.1}^{2}\right) ; 17$ loci were distributed over ten cultivated cardoon linkage groups, one (SNP site 5548175) was associated to a previously linked pairs of markers and thereby generated a new LG (Alt_22), and CAPS 14600-111 was linked to the previously unmapped microsatellite locus CyEM-134 (Figure 6). CAPS loci 5983-127 and 20149-154 were most tightly linked with one another (1.3 cM on LG Alt_1b+16). The inclusion of these 17 loci generated only minor changes in locus order; some rearrangements were induced in Alt_4 (CELMS-42, $\Delta 10.0$ cM), Alt_8 (CyEM_48, $\Delta 10.8$ cM and CyEM_286, $\Delta 11.2$ cM) and Alt_9 (e39/m50-240, $\Delta 19.4$ cM). The mapping exercise confirmed that the RAD-derived SNP markers are suitable for genotyping purposes.

\section{Conclusion}

In crop species where the number of markers available to date is limiting, the use of high throughput sequencing to generate large numbers of genetically informative assays can make a valuable and rapid contribution to linkage mapping, and its major downstream application, markerassisted selection. RAD tag sequencing based on the Illumina platform has proven to be a highly reliable and costeffective means of SNP discovery. We were able to identify thousands of putative SNP markers in this way, and the majority of a random sample of 24 was fully validated through conversion to CAPS assays and linkage analysis. Furthermore, the reduction in template complexity generated by the RAD approach greatly facilitates its implementation in mapping-by-sequencing approaches.

A large proportion of the methylation present in DNA occurs in the form of $\mathrm{CpG}$ dinucleotides, and there is little evidence for negative selection against these in the many genomes which have been analysed to date [32,37]. Acquiring genome-wide sequence has given a glimpse of the genome complexity present in $C$. cardunculus. Even though the RAD tags represent only a sample of the genome as a whole, it was clear that there exists a relationship between the frequency of $\mathrm{CpG}$ dinucleotides and the level of sequence repetitiveness, consistent with the known role played by methylation in controlling genome expansion due to transposable element activity [30,31].

\section{Methods}

Plant material and RAD tag sequencing

Genomic DNA was extracted from the leaf of the three C. cardunculus accessions, following the protocol described by Lanteri et al. [38]. The three accessions have been used as parents of two $F_{1}$ populations, made by crossing globe artichoke variety "Romanesco C3" as female with either the cultivated cardoon variety "Altilis 41 " or the wild cardoon accession "Creta 4" as male [11]. "Romanesco C3" is a late-maturing variety, which forms large purple-green capitula, each bearing violet coloured florets; "Altilis 41" was selected at the University of Catania [11] on the basis of its biomass yield potential; its foliage is grey and its florets white. "Creta 4" was collected from a wild population in Crete; it produces a large number of capitula, forms green-violet bracts and violet florets. Each DNA sample was processed into a separate RAD libraries as reported by Baird et al. [17]. Briefly, 300 ng DNA were digested with $20 \mathrm{U}$ of PstI (New England Biolabs, NEB) for $60 \mathrm{~min}$ at $37^{\circ} \mathrm{C}$ in a $50 \mu$ reaction, after which the reactions were heat inactivated by holding at $65^{\circ} \mathrm{C}$ for $20 \mathrm{~min}$. A $2.5 \mu \mathrm{L}$ aliquot of $100 \mathrm{nM}$ P1 adaptor (a modified Illumina adapter) [18] was added to each sample along with $1 \mu \mathrm{L}$ 10 mM ATP (Promega), $1 \mu \mathrm{L}$ 10x NEB Buffer4, 1,000 U T4 DNA ligase (Enzymatics, Inc) and $5 \mu \mathrm{L} \mathrm{H}_{2} \mathrm{O}$, and the reaction was incubated at room temperature for $20 \mathrm{~min}$, ending with a heat inactivation step $\left(65^{\circ} \mathrm{C} / 20\right.$ $\min )$. The reactions were then pooled and sheared to an average length of 500 bp using a Bioruptor (Diagenode). The sheared DNA was separated by electrophoresis through a $1.5 \%$ agarose gel, and fragments in the 300 800 bp range were isolated using a MinElute Gel Extraction kit (Qiagen). The End-Repair mix (Enzymatics, Inc.) was used to blunten the dsDNA ends, and the samples were re-purified using a MinElute column (Qiagen), following which $15 \mathrm{U}$ Exo-Klenow (Enzymatics, Inc.) were added and the sample incubated at $37^{\circ} \mathrm{C}$ to generate 3 'adenine overhangs. After subsequent purification, $1 \mu \mathrm{L}$ $10 \mu \mathrm{M}$ P2 adapter (a second modified Illumina adapter) [18] was ligated and the sample purified as above. The concentration of DNA in the eluate was quantified using a Qubit fluorimeter, and a $20 \mathrm{ng}$ aliquot was used for a $100 \mu \mathrm{L}$ PCR comprising $20 \mu \mathrm{L}$ Phusion Master Mix (NEB), $5 \mu \mathrm{L} 10 \mu \mathrm{M}$ P2 and $\mathrm{H}_{2} \mathrm{O}$. The 18 cycle PCR amplification regime followed the recommendation of the manufacturer (NEB). After this PCR, the samples were separated by electrophoresis once again through a $1.5 \%$ agarose gel, and fragments in the 300-700 bp range were excised from the gel and diluted to $3 \mathrm{ng} / \mu \mathrm{L}$. The material was analysed on an Illumina Genome Analyzer IIx following the paired ends (2x $54 \mathrm{bp}$ ) genomic DNA sequencing protocol suggested by the manufacturer.

\section{RAD contig assembly}

The sequences were sorted according to their multiplex identifier tag. A RAD LongRead ${ }^{\circledR}$ contig assembly was generated by a set of algorithms developed at Floragenex 


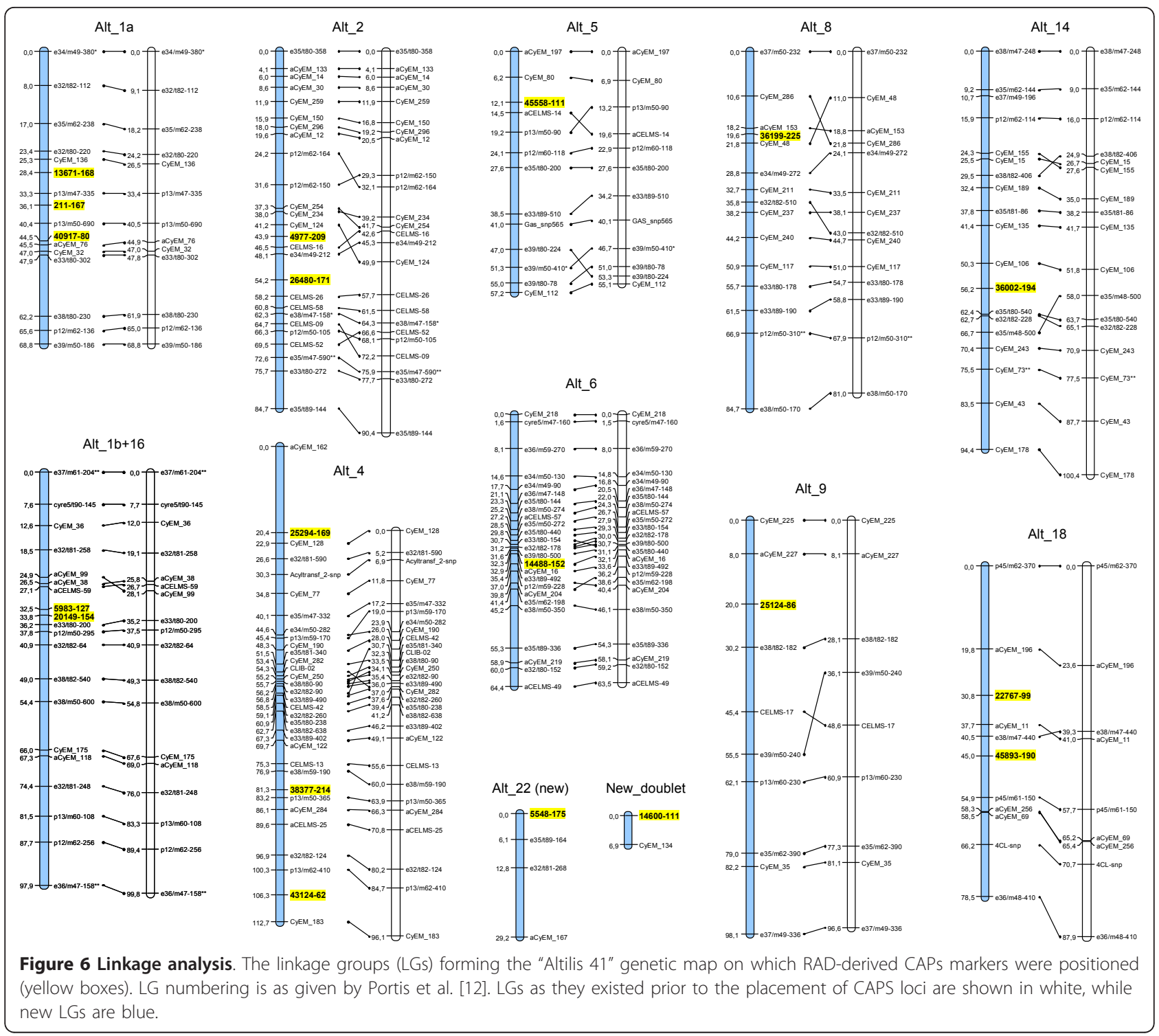

Inc. Sequences having more than 5 bases with poor Illumina quality scores (Phred10 or lower) were discarded. Paired reads were collapsed into sequence "clusters" on the basis of single ends (SE) sharing 100\% sequence identity. To maximize assembly efficiency, a minimum of $25 \mathrm{x}$ and maximum 400x sequence coverage at RAD SE reads were imposed. The variable paired end sequences for each common SE were extracted using the filtered sequence set and compiled for the LongRead ${ }^{\circledR}$ contig construction, using a modified version of the Velvet sequence assembler (v. 1.0.04) [39] and testing several kmers in graph construction for each RAD contig. After analysis of the first-pass assembly from each template, "Creta 4" was selected as the reference sequence set. Additional filters were then applied to remove short contigs $(<100 \mathrm{bp}$ in length), low paired end coverage
$(<4.0 x)$ or ambiguous contigs (containing N's homopolymers). If more than a single contig (NODE1) was assembled for a given RAD locus, alternative ones were retained in the dataset and labelled accordingly (NODE2, NODE3).

\section{Contig annotation and categorization}

RAD contigs were annotated using Blast2GO software [40], and were submitted to the NCBI nr protein database where an E-value of $10 \mathrm{e}^{-3}$ or lower were retrieved (20 best hits recorded). Gene names and GIs (gene identifiers) were assigned according to NCBI guidelines, and PIR (Protein Information Resource) identifiers in reference to UniProt, SwissProt, TrEMBL, RefSeq, GenPept and PDB. The annotation was obtained by applying the formula embedded in Blast2GO [40], setting a threshold 
score of 55. In the Blast2Go pipeline, GO terms are "transferred" to query sequences only whether a score threshold is reached. This score is calculated basing on both sequence similarity and presence of children node in the directed acyclic graph (DAG). Therefore, in this scenario the first e-value cut-off is used only for the purpose of "collecting" GO-terms, while other more stringent criteria are ruling whether transfer these terms to our sequences. Enzyme codes were retrieved from GO tables and mapped onto KEGG pathways. Transposable elements were detected using RepeatMasker v3.2.9 software http://www.repeatmasker.org, based on the RMBlast algorithm. Default parameters (except for -s flag) were used to search against Viridiplantae repeats.

\section{K-mer distribution and CpG suppression}

K-mer distribution and CpG suppression were analyzed using a Python script to split K-mer counts generated with Jellyfish [41]. The whole genome assemblies of $A$. thaliana, $V$. vinifera and $F$. vesca were retrieved from TAIR http://www.arabidopsis.org, PlantGDB http://www. plantgdb.org/VvGDB/ and PFR Strawberry server http:// www.strawberrygenome.org, respectively. For C. cardunculus, the K-mer distribution was generated using the raw paired end sequence of "Creta 4" and its de novo assembled contigs. K-mers of length $10 \mathrm{nt}$ were considered, and split according to the presence of $0,1,2$ or more CpG. The "CpG rate" was estimated according as proposed by Karlin and Mrazek [32]:

$$
p(C p G)=\frac{C p G}{p(C) p(G)}
$$

where $\mathrm{CpG}$ represents the observed frequency of $\mathrm{CpG}$ dinucleotides and $\mathrm{p}(\mathrm{C})$ and $\mathrm{p}(\mathrm{G})$ the respective frequencies of each single nucleotide.

\section{SNP discovery}

MAQ software (v. 0.5.0) [42] was used to align the paired end reads in the "Creta 4" reference contig set. The alignment threshold was set to a maximum of three nucleotides mismatch between Illumina reads and the reference. Gaps in the alignment of up to 2 nt allowed. Two levels of stringency were applied. In the first (CcRAD1), a comprehensive list of putative SNPs and 1-2 bp indels was populated with a minimum coverage of $6 \mathrm{x}$ as threshold prior to uploading to a Microsoft Access relational database; and for the second (CcRAD2), "fully informative" SNPs were defined when a minimum of 1-read allele calling was achieved for each of the three samples. In the latter set, heterozygous SNPs were assessed where the within sample allele frequency ranged from 0.25 to 0.75 , together with a minimum coverage of $4 \mathrm{x}$ and allele calling for two reads. Sites were assigned as homozygous when the minor allele frequency fell below 0.10 .
Candidate SNP markers were categorized as testcross in pair-wise comparisons of genotypes, whether a heterozygous imputation was present for one parent only (testcross) and a homozygous site was predicted for the other. Common intercross markers were defined for loci showing heterozygous states across all the three samples.

\section{CAPS assay design and application}

A subset of heterozygous SNPs was selected from the "Altilis 41" sequence, and a search carried out for BamHI, EcoRI, EcoRV, NdeI, XbaI, BccI, FokI, XmnI and DraI (6 bp cutters), or TaqI and MseI (4 bp cutters) recognition sites using SNP2CAPS script (v. 0.6) [43]. A predicted fragment size difference of at least $20 \mathrm{bp}$ was imposed to allow detection on standard agarose gels. Locus-specific primers were designed from the BatchPrimer3 web interface [44], using default parameters but for product size (100-400 bp) and annealing sites (within a $50 \mathrm{bp}$ window at either end of the RAD contig). The resulting assays were applied to a set of $94 \mathrm{~F}_{1}$ segregants from the cross "Romanesco C3" × "Altilis 41" [11]. PCRs were carried out in a $20 \mu \mathrm{l}$ volume containing $12.5 \mathrm{ng}$ genomic DNA, $1 \mathrm{x}$ GoTaq Buffer (Promega), $1.5 \mathrm{mM} \mathrm{MgCl} 2,0.2 \mathrm{mM}$ dNTPs, $1 \mathrm{U}$ GoTaq (Promega) and $0.5 \mu \mathrm{M}$ of each primer. The cycling regime was $95^{\circ} \mathrm{C} / 5 \mathrm{~min}$, followed by 35 cycles of $95^{\circ} \mathrm{C} / 30 \mathrm{~s}, 55^{\circ} \mathrm{C} / 30 \mathrm{~s}, 72^{\circ} \mathrm{C} / 45 \mathrm{~s}$ and a final incubation of $72^{\circ} \mathrm{C} / 5 \mathrm{~min}$. Amplification was checked by electrophoresis through a $1.5 \%$ agarose gel and quantified using a Beckman Coulter spectrophotometer. Restriction reactions $(20 \mu \mathrm{l})$ comprised $800 \mathrm{ng}$ amplified DNA, $0.3 \mathrm{U}$ restriction enzyme (New England Biolabs), reaction buffer and BSA according to the manufacturers' specifications, incubated for $4 \mathrm{~h}$ at $37^{\circ} \mathrm{C}$ (except for $\mathrm{TaqI}$, where the incubation temperature was $65^{\circ} \mathrm{C}$ ), after which the reactions were heat inactivated $\left(80^{\circ} \mathrm{C} / 10 \mathrm{~min}\right)$. The resulting products were electrophoresed through $2 \%$ agarose gels.

\section{Linkage analysis}

The CAPS derived genotypic data were incorporated into a pre-existing data set of 273 molecular loci, mainly AFLP and EST-SSRs, already used to generate the cultivated cardoon genetic map $[11,14,15]$ including five SNP from genes underlying caffeoylquinic acids synthesis reported by Comino et al. [45] and Menin et al. [46]; all maps data are available on request by the authors. Goodness-of-fit between observed and expected segregation ratios was tested by $\chi^{2}$ and only markers fitting or deviating only marginally from expectation $\left(\chi_{\alpha=1}^{2}<\chi^{2} \leq \chi_{\alpha=0.01}^{2}\right)$ were included for mapping. Linkage groups (LGs) were established by JoinMap v4.0 software [47], on the basis of a LOD threshold of 6.0, using as parameter settings Rec = $0.40, \mathrm{LOD}=1.0$, Jump $=5$. Map distances were converted to centiMorgans (cM) using the Kosambi mapping 
function. LGs were drawn and aligned using MapChart v2.1 [48].

\section{Additional material}

\section{Additional file 1: The data provided represent the assembled RAD} contigs in fasta format. RAD contigs.

Additional file 2: The data provided represent the list of the RAD contigs which were annotated with the Blast2Go pipeline. Contigs annotation.

Additional file 3: The data provided represent the list of the RAD contigs which were mapped in the KEGG's pathway. KEGG's pathways mapping.

Additional file 4: Distributions of K-mers using random dinucleotides. distributions of $\mathrm{K}$-mers.

Additional file 5: Comprehensive list of SNPs and 1-2 bp indels. CCRAD1 SNP list.

Additional file 6: list of the "fully informative" SNPs and test cross markers. CCRAD2 SNP list.

\section{Acknowledgements}

This research was supported by grants from: (i) the National Science Foundation Plant Genome Research Program (No. 0421630), (ii) the Georgia Research Alliance, (iii) the University of Georgia Research Foundation, and (iv) by MIPAAF (Ministero delle Politiche Agricole, Alimentari e Forestali Italy) through the CYNERGIA ("Costituzione e valutazione dell'adattabilita' di genotipi di Cynara cardunculus per la produzione di biomassa e biodiesel in ambiente mediterraneo") project and CARVARVI ("Valorizzazione di germoplasma di carciofo attraverso la costituzione varietale ed il risanamento da virus") project.

\section{Author details}

'Di.Va.P.R.A. Plant Genetics and Breeding, University of Torino, via L. da Vinci 44, 10095 Grugliasco (Torino), Italy. ${ }^{2}$ Institute for Plant Breeding, Genetics, and Genomics, University of Georgia, 111 Riverbend Rd., 30602 Athens, Georgia USA.

\section{Authors' contributions}

SK and SL planned and supervised the experimental work; DS carried out the bioinformatic analysis; DS and MT performed the genotyping of the progenies; EP carried out linkage analyses and map construction; AA and EP and SL supervised the drafting of the manuscript. All authors read and approved the final manuscript.

Received: 6 August 2011 Accepted: 3 January 2012

Published: 3 January 2012

\section{References}

1. Marie D, Brown S: A cytometric exercise in plant DNA histograms, with 2C values for 70 species. Biol Cell 1993, 78:41-51.

2. Rottenberg A, Zohary D: The wild ancestry of the cultivated artichoke. Genet Resour Crop Ev 1996, 43(1):53-58.

3. Shimoda H, Ninomiya K, Nishida N, Yoshino T, Morikawa T, Matsuda H, Yoshikawa M: Anti-hyperlipidemic Sesquiterpenes and new sesquiterpene glycosides from the leaves of artichoke (Cynara scolymus L.): Structure requirement and mode of action. Bioorg Med Chem Lett 2003, 13(2):223-228.

4. Gebhardt R: Antioxidative and protective properties of extracts from leaves of the artichoke (Cynara scolymus $\mathrm{L}$ ) against hydroperoxideinduced oxidative stress in cultured rat hepatocytes. Toxicol Appl Pharm 1997, 144(2):279-286.

5. Gebhardt R: Inhibition of cholesterol biosynthesis in HepG2 cells by artichoke extracts is reinforced by glucosidase pretreatment. Phytother Res 2002, 16(4):368-372.
6. Schutz K, Kammerer D, Carle R, Schieber A: Identification and quantification of caffeoylquinic acids and flavonolds from artichoke (Cynara scolymus L.) heads, juice, and pomace by HPLC-DAD-ESI/MSn. J Agr Food Chem 2004, 52(13):4090-4096.

7. Ierna A, Mauromicale G: Cynara cardunculus L. genotypes as a crop for energy purposes in a Mediterranean environment. Biomass Bioenerg 2010, 34(5):754-760.

8. Raccuia S, Melilli M: Biomass and grain oil yields in Cynara cardunculus L. genotypes grown in a Mediterranean environment. Field Crop Res 2007, 101(2):187-197.

9. Encinar J, Gonzalez J, Rodriguez J, Tejedor A: Biodiesel fuels from vegetable oils: Transesterification of Cynara cardunculus L. oils with ethanol. Energ Fuel 2002, 16(2):443-450.

10. Encinar J, Gonzalez J, Gonzalez J: Steam gasification of Cynara cardunculus L.: influence of variables. Fuel Proces Technol 2002, 75(1):27-43.

11. Lanteri S, Acquadro A, Comino C, Mauro R, Mauromicale G, Portis E: A first linkage map of globe artichoke (Cynara cardunculus var. scolymus $\mathrm{L}$.) based on AFLP, S-SAP, M-AFLP and microsatellite markers. Theor Appl Genet 2006, 112(8):1532-1542.

12. Portis E, Mauromicale G, Mauro R, Acquadro A, Scaglione D, Lanteri S: Construction of a reference molecular linkage map of globe artichoke (Cynara cardunculus var. scolymus). Theor Appl Genet 2009, 120(1):59-70.

13. Sonnante G, Gatto A, Morgese A, Montemurro F, Sarli G, Blanco E, Pignone D: Genetic map of artichoke $\times$ wild cardoon: toward a consensus map for Cynara cardunculus. Theor Appl Genet 2011, 123(7):1215-1229.

14. Scaglione D, Acquadro A, Portis E, Taylor C, Lanteri S, Knapp S: Ontology and diversity of transcript-associated microsatellites mined from a globe artichoke EST database. BMC Genomics 2009, 10:454

15. Portis E, Acquadro A, Scaglione D, Comino C, Mauromicale G, Mauro R, Taylor C, Knapp S, Lanteri S: Construction of a reference linkage map for globe artichoke. Proceedings of the 53rd Italian Society of Agricultural Genetics Annual Congress Torino, Italy - 16/19 September 2009 [http://www. siga.unina.it/SIGA2009/SIGA_2009/1_04.pdf], ISBN 978-88-900622-9-2.

16. Portis E, Acquadro A, Scaglione D, Mauromicale G, Mauro R, Taylor CA, Knapp SJ, Lanteri S: Construction of an SSR-based linkage map for Cynara cardunculus. 2009, 8th Plant Genomics European Meeting.

17. Miller M, Dunham J, Amores A, Cresko W, Johnson E: Rapid and costeffective polymorphism identification and genotyping using restriction site associated DNA (RAD) markers. Genome Res 2007, 17:240-248.

18. Baird N, Etter P, Atwood T, Currey M, Shiver A, Lewis Z, Selker E, Cresko W, Johnson E: Rapid SNP Discovery and Genetic Mapping Using Sequenced RAD Markers. Plos One 2008, 3(10):e3376.

19. Pfender WF, Saha MC, Johnson EA, Slabaugh EA: Mapping with RAD (restriction-site associated DNA) markers to rapidly identify QTL for stem rust resistance in Lolium perenne. Theor Appl Genet 2011, 122(8):1467-1480.

20. Chutimanitsakun Y, Nipper R, Cuesta-Marcos A, Cistue L, Corey A, Filichkina T, Johnson E, Hayes P: Construction and application for QTL analysis of a Restriction Site Associated DNA (RAD) linkage map in barley. BMC Genomics 2011, 12:4.

21. Barchi L, Lanteri S, Portis E, Acquadro A, Vale G, Toppino L, Rotino GL: Identification of SNP and SSR markers in eggplant using RAD tag sequencing. BMC Genomics 2011, 12:304.

22. Hohenlohe P, Amish S, Catchen J, Allendorf F, Luikart G: Next-generation RAD sequencing identifies thousands of SNPs for assessing hybridization between rainbow and westslope cutthroat trout. Mol Ecol Res 2011, 11:117-122.

23. Kaul S, Koo H, Jenkins J, Rizzo M, Rooney T, Tallon L, Feldblyum T, Nierman W, Benito $M$, Lin $X$, et al: Analysis of the genome sequence of the flowering plant Arabidopsis thaliana. Nature 2000, 408:796-815.

24. Jaillon O, Aury J, Noel B, Policriti A, Clepet C, Casagrande A, Choisne N, Aubourg S, Vitulo N, Jubin $C$, et al: The grapevine genome sequence suggests ancestral hexaploidization in major angiosperm phyla. Nature 2007, 449:463-467.

25. Etter PD, Preston JL, Bassham S, Cresko WA, Johnson EA: Local De Novo Assembly of RAD Paired-End Contigs Using Short Sequencing Reads. Plos One 2011, 6(4):e18561.

26. Baxter SW, Davey JW, Johnston JS, Shelton AM, Heckel DG, et al: Linkage Mapping and Comparative Genomics Using Next-Generation RAD Sequencing of a Non-Model Organism. PLoS One 2011, 6(4):e19315. 
27. Willing EM, Hoffmann M, Klein JD, Weigel D, Dreyer C: Paired-end RAD-seq for de-novo assembly and marker design without available reference. Bioinformatics 2011, 27(16):2187-2193.

28. Palmer LE, Rabinowicz PD, O'Shaughnessy AL, Balija VS, Nascimento LU, Dike S, de la Bastide M, Martienssen RA, McCombie WR: Maize Genome Sequencing by Methylation Filtration. Science 2003, 302(5653):2115-2117.

29. Chor B, Horn D, Goldman N, Levy Y, Massingham T: Genomic DNA k-mer spectra: models and modalities. Genome Biol 2009, 10:108.

30. Slotkin R, Vaughn M, Borges F, Tanurdzic M, Becker J, Feijo J, Martienssen R: Epigenetic reprogramming and small RNA silencing of transposable elements in pollen. Cell 2009, 136(3):461-472

31. Lisch D: Epigenetic regulation of transposable elements in plants. Annual Review of Plant Biology 2009, 60:43-66.

32. Karlin S, Mrazek J: Compositional differences within and between eukaryotic genomes. P Natl Acad Sci USA 1997, 94(19):10227-10232.

33. Shulaev V, Sargent D, Crowhurst R, Mockler T, Folkerts O, Delcher A Jaiswal P, Mockaitis K, Liston A, Mane S, et al: The genome of woodland strawberry (Fragaria vesca). Nat Genet 2011, 43:109-116.

34. Velasco R, Zharkikh A, Troggio M, Cartwright D, Cestaro A, Pruss D, Pindo M, FitzGerald L, Vezzulli S, Reid J, et al: A high quality draft consensus sequence of the genome of a heterozygous grapevine variety. Plos One 2007, 2(12):e1326.

35. Jiang $D, Y e Q$, Wang F, Cao L: The mining of citrus EST-SNP and its application in cultivar discrimination. Agricultural Sciences in China 2010, 9(2):179-190.

36. Portis E, Mauromicale G, Barchi L, Mauro R, Lanteri S: Population structure and genetic variation in autochthonous globe artichoke germplasm from Sicily Island. Plant Sci 2005, 168(6):1591-1598.

37. Stacey K, Young G, Clark F, Sester D, Roberts T, Naik S, Sweet M, Hume D: The molecular basis for the lack of immunostimulatory activity of vertebrate DNA. J Immunol 2003, 170:3614-3620.

38. Lanteri S, Di Leo I, Ledda L, Mameli M, Portis E: RAPD variation within and among populations of globe artichoke cultivar 'Spinoso sardo'. Plant Breeding 2001, 120(3):243-246.

39. Zerbino D, Birney E: Velvet: Algorithms for de novo short read assembly using de Bruijn graphs. Genome Res 2008, 18:821-829.

40. Conesa A, Gotz S, Garcia-Gomez J, Terol J, Talon M, Robles M: Blast2GO: a universal tool for annotation, visualization and analysis in functional genomics research. Bioinformatics 2005, 21(18):3674-3676.

41. Marcais $G$, Kingsford C: A fast, lock-free approach for efficient parallel counting of occurrences of k-mers. Bioinformatics 2011, 27(6):764-770.

42. Li H, Ruan J, Durbin R: Mapping short DNA sequencing reads and calling variants using mapping quality scores. Genome Res 2008, 18(11):1851-1858.

43. Thiel T, Kota R, Grosse I, Stein N, Graner A: SNP2CAPS: a SNP and INDEL analysis tool for CAPS marker development. Nucleic Acids Res 2004, 32(1): e5.

44. You F, Huo N, Gu Y, Luo M, Ma Y, Hane D, Lazo G, Dvorak J, Anderson O: BatchPrimer3: A high throughput web application for PCR and sequencing primer design. Bmc Bioinformatics 2008, 9:253.

45. Comino C, Hehn A, Moglia A, Menin B, Bourgaud F, Lanteri S, Portis E: The isolation and mapping of a novel hydroxycinnamoyltransferase in the globe artichoke chlorogenic acid pathway. BMC Plant Biol 2009, 9:30

46. Menin B, Comino C, Moglia A, Dolzhenko Y, Portis E, Lanteri S: Identification and mapping of genes related to caffeoylquinic acid synthesis in Cynara cardunculus L. Plant Sci 2010, 179(4):338-347.

47. Van O: JoinMap ${ }^{\circledR}$ 4: software for the calculation of genetic linkage maps in experimental populations. Wageningen: Kyazma B.V; 2006.

48. Voorrips R: MapChart: Software for the graphical presentation of linkage maps and QTLs. J Hered 2002, 93(1):77-78.

doi:10.1186/1471-2164-13-3

Cite this article as: Scaglione et al:: RAD tag sequencing as a source of SNP markers in Cynara cardunculus L. BMC Genomics 2012 13:3.

\section{Submit your next manuscript to BioMed Central and take full advantage of:}

- Convenient online submission

- Thorough peer review

- No space constraints or color figure charges

- Immediate publication on acceptance

- Inclusion in PubMed, CAS, Scopus and Google Scholar

- Research which is freely available for redistribution

Submit your manuscript at www.biomedcentral.com/submit
C) Biomed Central 